of controls, and the dexamethasone group may have been less severely ill than the controls. An assessment of higher cortical function one year after discharge revealed no significant difference in treatment and control groups. Dexamethasone in the treatment of a severely ill child with meningitis may be recommended but a confirmatory study documenting safety is advised to determine its necessity in mild cases. Dexamethasone may save the hearing but worsen cerebal cortical function by ischemic injury and may induce gastrointestinal bleeding.

\title{
INTRACRANIAL TUBERCULOMA
}

In a report from the Dept of Pediatrics, All India Inst of Applied Sciences, New Delhi, India, intracranial tuberculoma was found in $20(24 \%)$ of 83 patients with partial seizures complicated by increased intracranial pressure, systemic tuberculosis or focal neurologic deficit and in $12(22 \%)$ of 55 patients with tuberculous meningitis. CT lesions consisting of ring enhancement, discs, and irregular coalescing masses with edema regressed within 12 weeks of starting medical therapy. Surgical excision was not required. Medical management was advocated, with surgery limited to drug treatment failures. (Bagga A et al. Intracranial tuberculoma. Evaluation and treatment. Clin Pediat Oct 1988;27:487-490).

COMMENT. Tuberculoma mimics other space-occupying lesions and in India its reported prevalence has ranged from 4 to $40 \%$ of intracranial tumors. Effective chemotherapy and CT monitoring of treatment response have minimized the role of surgery.

\section{INTRACRANIAL TUMORS}

RADIATION-INDUCED TUMORS

The relation between radiotherapy to the head and neck for tinea capitis childhood and the later development of tumors of the brain and nervous system have been investigated in 10,834 patients treated between 1948 and 1960 in Israel and the results evaluated at the Radiation Epidemiology Branch, National Cancer Institute, Bethesda, MD. Neural tumors developed in 73 patients, 60 in irradiated subjects, 8 among general population controls, and 5 among sibling controls. The increase in incidence among those irradiated was 7 times that of controls. The relative risk of all head and neck situated neural tumors among irradiated subjects was 8.4 . Increased relative risks were greater for benign nerve-sheath tumors $(18.8 ; n=25)$ than for meningiomas $(9.5 ; n=19)$ and gliomas $(2.6 ; n=7)$. A strong doseresponse relation was shown, the risk approaching 20 after doses of $2.5 \mathrm{~Gy}$. Radiation doses to the head and neck in childhood on the order of 1 to 2 GY significantly increased the risk of neural tumors in those areas. (Ron $E$ et al Tumors of the brain and nervous system after radiotherapy in childhood. $N$ Engl J Med Oct 20 1988; 319:1033-9). 
COMMENT. Radiation-induced tumors of the central nervous system are recognized as a consequence of combined treatments for leukemia chemotherapy and irradiation to the head. Therapeutic doses of radiation for childhood leukemia are higher than those used for the patients with tinea capitis in Israel and the relative risks are undetermined.

\section{BEHAVIOR AND LEARNING DISABILITIES}

ATTENTION DEFICIT DISORDER

The go-no-go paradigm was used in the evaluation of children with attention deficit disorder (ADD) at the Dept of Pediatrics, Division of Neurology and Evaluation Center for Learning, Northwestern Univ Medical School, Evanston Hospital, Evanston, IL. The paradigm consisted of the taped presentation of 2 trials of 10 stimuli; 5 go signals (1 tap) to which the children were expected to respond by raising and lowering their index finger, and 5 no-go signals to which they should not respond. Commissions errors suggest impulsivity and omission errors suggest inattention. Children with ADD ( 44 boys) made more total errors than did 32 control subjects $(p<.03)$. Nonhyperactive (ADDnoH) subjects made more commission errors than controls initially but improved with practice. Hyperactive ADD subjects (ADDH) made the same number of early commission errors as controls but failed to improve with practice. Omission errors were highest in the ADDH group. The paradigm provided an objective measure of inattention and impulsivity and a distinction between hyperactive and nonhyperactive children with ADD.: (Trommer Bl et al. The go-no-go paradigm in attention deficit disorder. Ann Neurol Nov 1988;24:610-614).

COMMENT. Errors of commission are more common than errors of omission in children with ADD which suggests that impulsivity is more easily demonstrated than inattention. The DSM-III "impulsivity" criteria include inability to wait in turn at games, calling out in class, and shifting activities. The go-no-go paradigm offers a test for impulsivity at a cognitive level, the inability to give the most correct answer in a multiple choice setting, and supplements reports of behavioral manifestations of ADD.

\section{FLOATING-HARBOR SYNDROME}

Six unrelated children with a unique association of short stature, dysmorphic features, and speech delay are reported from the Harbor/Univ of California at Los Angeles Med Center, the Kennedy Memorial Hospital, Boston, the Beilinson Med Center, the District General Hospital, Stanford, England, and the CedarsSinai Med Cntr, Univ of California, LA. Two were FrenchCanadian, 2 British, 1 Iranian, and 1 Israeli ancestry. All had growth retardation during the first year, delayed bone age, severe speech delay and normal or only mildly retarded intelligence. The strikingly similar facial features consisted 